VOL. 41 (1990) [117-122]

\title{
NOTE ON THE EXTENSIONS OF BUTLER GROUPS
}

\section{FuChS AND G. VILJOEN}

In terms of a new notion, called prebalancedness, those extensions of a Butler group by a Butler group which are again Butler groups are characterised. It is shown that the prebalanced extensions of torsion-free abelian groups form a divisible subgroup in the group of extensions.

\section{INTRODUCTION}

A lot of current research in the theory of torsion-free abelian groups is focussed on so-called Butler groups. Butler groups are defined either as pure subgroups of, or, (equivalently) as torsion-free epic images of finite rank completely decomposable torsion-free groups: see Arnold [2]. They form an attractive class rich in challenging problems.

The class of Butler groups is closed under finite direct sums as well as under the formations of pure subgroups and torsion-free epic images. It is not closed under extensions (otherwise all finite rank torsion-free groups would be Butler groups), and therefore it seems to be natural to enquire which extensions of Butler groups are again Butler groups.

An answer to this question may be given in terms of 'decent' subgroups introduced by Albrecht and Hill [1]. However, 'decency' is the combination of two simpler, more natural concepts (Theorem 8 ) one of which leads to a more direct answer to our question. This concept reflects a property of subgroups which is weaker than balancedness or decency; we call it prebalancedness. We will show that an extension $A$ of a Butler group $B$ by a Butler group $C$ is again Butler if and only if $B$ is prebalanced in $A$ (Theorem 5). This property is intimately related to Butler groups: Butler groups can be characterised as those finite rank torsion-free groups in which all pure subgroups are prebalanced.

Giovannitti [4] proved that the extensions of a Butler group $B$ by a Butler group $C$ which are again Butler groups form a divisible subgroup in $\operatorname{Ext}(C, B)$. We generalise this by showing that the prebalanced extensions of torsion-free groups form a divisible subgroup of the group of all extensions which contains the torsion part (see Theorem 4).

Received 22 February, 1989

The first author was partially supported by NSF grant DMS-8620379. The second author was supported through grants by the C.S.I.R.(F.R.D.) and the University of Orange Free State.

Copyright Clearance Centre, Inc. Serial-fee code: 0004-9729/90 \$A2.00+0.00. 


\section{Prebalanced subgroups}

All groups under consideration are additively written torsion-free abelian groups. For unexplained terminology and notation we refer to [3].

We start with the definition which generalises the familiar concept of balancedness for torsion-free groups (see[3, Vol. 2, p.113]).

Let $B$ be a pure subgroup of a torsion-free group $A$. It will be called prebalanced in $A$ if for every $a \in A$ there is a finite subset $\left\{b_{1}, \ldots, b_{n}\right\} \subseteq B$ such that

$$
\chi_{A / B}(a+B)=\chi_{A}\left(a+b_{1}\right) \cup \ldots \cup \chi_{A}\left(a+b_{n}\right) .
$$

Here $\chi_{A}$ denotes the characteristic of an element in $A$. Balancedness corresponds to the case when $n=1$ can always be chosen. If $B$ is prebalanced in $A$, we say the exact sequence $0 \rightarrow B \rightarrow A \rightarrow A / B \rightarrow 0$ is prebalanced-exact.

The following lemma is crucial in dealing with this concept.

LEмMA 1. For a pure subgroup $B$ of the torsion-free group $A, a \in A,\left\{b_{1}, \ldots, b_{n}\right\}$ $\subseteq B$ satisfy (1) if and only if

$$
\langle B, a\rangle_{\star}=B+\left\langle a+b_{1}\right\rangle_{\star}+\ldots+\left\langle a+b_{n}\right\rangle_{\star} .
$$

Proof: We shall use the notations $A_{i}=\left\langle a+b_{i}\right\rangle_{\star}$ and $C=\langle B, a\rangle_{\star}$ where $\star$ indicates purification.

First, suppose (1). Then $B+A_{1}+\ldots+A_{n} \leqslant C$ is obvious. Assume $r(a+b) \in C$ for $r \in \mathbb{Q}$ and $b \in B$. Without loss of generality, we may restrict the proof to the case $r=$ $p^{-k}$ for a prime $p$ and an integer $k>0$. By (1), $p^{k} \mid a+B$ implies $p^{k} \mid a+b_{i}$ for some $i \in\{1, \ldots, n\}$, that is, $p^{-k}\left(a+b_{i}\right) \in A_{i}$. Therefore, from $p^{-k}\left(a+b_{i}\right)-p^{-k}(a+b) \in B$ we obtain $p^{-k}(a+b) \in B+A_{1}+\ldots+A_{n}$, and (2) holds.

Conversely, assume (2). It suffices to verify the inequality $\leqslant$ in (1). Form the outer direct sum $D=B \oplus A_{1} \oplus \ldots \oplus A_{n}$. The obvious map $\alpha: D \rightarrow C$ followed by the natural projection $\nu: C \rightarrow C / B$ yields an epimorphism $\nu \alpha: D \rightarrow C / B$. If $a+B$ is divisible by $p^{k}$ ( $p$ a prime and $k>0$ an integer), then there must exist a preimage $d=b+\Sigma n_{i}\left(a+b_{i}\right) \in D$ of $a+B$ under $\nu \alpha$ which is divisible by $p^{k} ;$ here $b \in B$, $n_{i} \in \mathbf{Q}$. Hence $p^{k} \mid a$ and $p^{k} \mid n_{i}\left(a+b_{i}\right)$ for each $i$ and, since $\nu \alpha d=a+B, \Sigma n_{i}=1$. Clearly, for the proof we may assume that all the $n_{i}$ 's are integers. As $\Sigma n_{i}=1$ implies that $p \nmid n_{j}$ for some index $j \in\{1, \ldots, n\}$, and thus $p^{k} \mid a+b_{j}$ for this $j$, the desired inequality follows.

Notice that (1) can be rephrased by stating that the rank one pure subgroup $C / B$ of $A / B$ is the sum of the images of $A_{1}, \ldots, A_{n}$ under $\nu$. Thus we have:

Corollary 2. A pure-exact sequence

$$
0 \rightarrow B \rightarrow A \stackrel{\alpha}{\rightarrow} C \rightarrow 0
$$


is prebalanced if and only if, for every rank 1 pure subgroup $J$ of $C$, there are a finite rank completely decomposable group $X=X_{1} \oplus \ldots \oplus X_{n}\left(X_{i}\right.$ of rank 1$)$ and an epimorphism $\xi: X \rightarrow J$ which lifts to a homomorphism $\eta: X \rightarrow A$ (that is, $\alpha \eta=\xi$ ).

The concept of prebalancedness looks worth further study. But such a study would go beyond our current goals, so just let us point out two elementary properties of prebalancedness which are needed in the next theorem.

LEMma 3. Consider the commutative diagram with exact rows where the middle row is prebalanced exact:

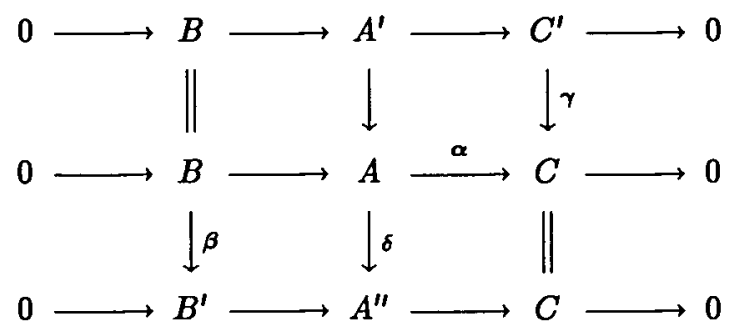

Then

(a) the bot tom row is prebalanced-exact;

(b) if $\gamma$ is monic, then the top row is prebalanced-exact.

Proof: To verify (a), let $J$ be a rank 1 pure subgroup of $C$. By Corollary 2, there exist $X=X_{1} \oplus \ldots \oplus X_{n}$ ( $\mathrm{rk} X_{i}=1$ ) and an epimorphism $\xi: X \rightarrow J$ which lifts to $\eta: X \rightarrow A$. This $\eta$ followed by $\delta$ is a desired lifting $X \rightarrow A^{\prime \prime}$.

(b) is evident, since $A^{\prime}$ may be viewed as a subgroup of $A$, and if $B$ is prebalanced in $A$, then it is prebalanced in the subgroup $A^{\prime}$ of $A$.

\section{ThE GROUP PBext.}

It is well-known that for a torsion-free group $C, \operatorname{Ext}(C, B)$ is a divisible group. We denote by $\operatorname{PBext}(C, B)$ the subset of $\operatorname{Ext}(C, B)$ consisting of those [equivalence classes of] extensions of $B$ by. $C$ which are represented by prebalanced-exact sequences $0 \rightarrow B \rightarrow A \rightarrow C \rightarrow 0$. This subset turns out to be a subgroup; moreover, we have the following generalisations of a result by Giovannitti [4]:

Theorem 4. For any torsion-free groups $B$ and $C, \operatorname{PBext}(C, B)$ is a divisible subgroup of $\operatorname{Ext}(C, B)$ which contains the torsion part of $\operatorname{Ext}(C, B)$.

Proof: Let

$$
E_{i}: 0 \rightarrow B \rightarrow A_{i} \rightarrow C \rightarrow 0 \quad(i=1,2)
$$

be prebalanced-exact sequences. In order to form their Baer sum, we consider the following commutative diagram with exact rows ( $\nabla$ and $\Delta$ are the diagonal and codiagonal 
maps, respectively):

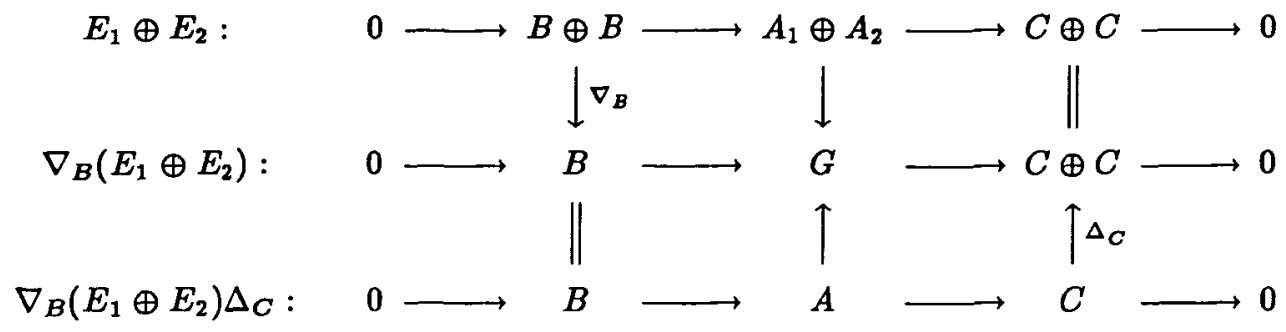

Manifestly, $E_{1} \oplus E_{2}$ is prebalanced-exact. Applying (a) and (b) of Lemma 3, we conclude that the second and third rows are prebalanced-exact. The bottom row is precisely $E_{1}+E_{2}$. Using Lemma 3(b) again (with $\gamma=-1_{C}$ ) we infer that $-E_{1}$ is likewise prebalanced-exact. The subgroup property of $\operatorname{PBext}(C, B)$ is established.

To complete the proof, it suffices to show that if $m E \in \operatorname{PBext}(C, B)$ for some $E \in \operatorname{Ext}(C, B)$ and integer $m>0$, then $E \in \mathrm{PBext}(C, B)$. Consider the commutative diagram with exact rows:

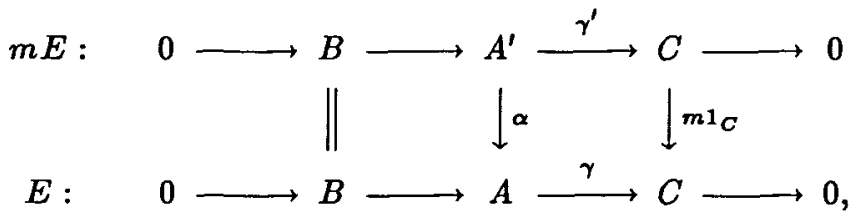

and suppose that the top row is prebalanced-exact. Let $J$ be a rank 1 pure subgroup of $C$. Using Corollary 3 , we can find a completely decomposable group $X=X_{1} \oplus \ldots \oplus X_{n}$ ( rk $X_{i}=1$ ) and an epimorphism $\xi: X \rightarrow J$ such that $\gamma^{\prime} \eta=\xi$ for some $\eta: X \rightarrow A^{\prime}$. Now $\gamma \alpha \xi$ maps $X$ onto $m J$ which is of finite index in $J$. Thus, using at most $m$ copies $Z_{j}$ of $Z$, we can extend $\gamma \alpha \xi: X \rightarrow m J$ to a surjective map $X \oplus Z_{1} \oplus \ldots \oplus Z_{k} \rightarrow J$. This map evidently lifts to $X \oplus Z_{1} \oplus \ldots \oplus Z_{k} \rightarrow A$, proving the prebalanced-exactness of $E$.

Giovannitti [4] investigates, for Butler groups $B, C$, certain subgroups of $\operatorname{PBext}(C, B)$ which are defined in terms of lattices of types.

\section{EXTENSIONS WHICH ARE BUTLER GROUPS}

We now concentrate on Butler groups and their extensions, and turn our attention to one of our main results.

TheOREM 5. Let

$$
0 \rightarrow B \rightarrow A \stackrel{\nu}{\rightarrow} C \rightarrow 0
$$


be an exact sequence of torsion-free groups of finite rank. $A$ is a Butler group if and only if

(i) both $B$ and $C$ are Butler groups; and

(ii) $B$ is prebalanced in $A$.

Proof: Suppose that $A$ is a Butler group. Then (i) is evident. Let $a \in B \backslash A$ and $A^{\prime}=\langle B, a\rangle_{\star}$. As a pure subgroup of a Butler group, $A^{\prime}$ is a Butler group, whence $A^{\prime}=A_{1}+\ldots+A_{n}$ for certain rank 1 subgroups $A_{i}$ of $A$. Thus $A^{\prime}=B+A_{1}+\ldots+A_{n}$ shows, by Lemma 1 , that (ii) holds (the $A_{i}$ with $A_{i} \leqslant B$ can be dropped).

Conversely, let (i) and (ii) hold. We can choose rank 1 pure subgroups $C_{i}$ in $C$ such that $C=C_{1}+\ldots+C_{m}$. By Lemma $1, D_{i}=\nu^{-1} C_{i}=B+A_{i 1}+\ldots+A_{i k_{i}}$ with rank 1 subgroups $A_{i j}$ of $A$. Hence $A=B+\sum_{i=1}^{m}\left(A_{i 1}+\ldots+A_{i k_{i}}\right)$. Here $B=B_{1}+\ldots+B_{k}$ with each $B_{i}$ a rank 1 subgroup of $B$; therefore $A$ is a Butler group.

Observe that the last result implies that in a Butler group all pure subgroups are prebalanced. Moreover, we have:

CoROLlary 6. A torsion-free group of finite rank is a Butler group exactly if all of its pure subgroups are prebalanced.

Proof: To establish sufficiency, let $A$ be torsion-free of finite rank $n$ such that all of its pure subgroups are prebalanced. There is a chain of pure subgroups, $0=$ $A_{0}<A_{1}<\ldots<A_{n}=A$ where the factors $A_{i} / A_{i-1}$ are of rank 1 . As rank 1 groups are Butler, it follows from Theorem 5 successively that $A_{1}, A_{2}, \ldots, A_{n}$ are Butler groups.

Warfield [5, p.140] proved that if $B, C$ are torsion-free groups of finite rank, then either $\operatorname{Ext}(C, B)=0$ or there are $2^{\aleph_{0}}$ non-quasi-isomorphic extensions $A$ of $B$ by $C$. Giovannitti [4] has shown that $\operatorname{PBext}(Q, Z)$ has the cardinality of the continuum, while $\operatorname{PBext}\left(Q, Z_{p} \cap Z_{q}\right)$ is countable for primes $p \neq q\left(Z_{p}\right.$ is the localisation of $Z$ at $\left.p\right)$.

\section{DECENT SUBGROUPS}

The notion of a decent subgroup has been introduced by Albrecht and Hill [1] in their study of infinite Butler groups. We wish to point out that prebalancedness generalises this concept.

A pure subgroup $B$ of a torsion-free group $A$ is called decent $[1, \mathrm{p} .302]$ if for every finite subset $S$ of $A$ there exist a finite number of rank 1 pure subgroups $A_{i}$ of $A$ such that $B+\sum A_{i}$ is pure in $A$ and contains $S$.

We shall call a torsion-free group $G$ locally Butler if every finite subset of $G$ can be embedded in a pure Butler subgroup of $G$. We can now characterise decency as follows. 
Theorem 8. A pure subgroup $B$ of a torsion-free group $A$ is decent if and only if (i) $B$ is prebalanced in $A$, and (ii) $A / B$ is a locally Butler group.

Proof: Let $B$ be decent in $A$ and $a \in A \backslash B$. There are pure rank 1 subgroups $A_{1}, \ldots, A_{k}$ of $A$ such that $a \in B+A_{1}+\ldots+A_{k}=A^{\prime}$ with $A^{\prime}$ pure in $A$. Then $C=A_{1}+\ldots+A_{k}$ is a Butler group, and by the modular law $\langle B, a\rangle_{\star}=B+\left(C \cap\langle B, a\rangle_{\star}\right)$. Here $C \cap\langle B, a\rangle_{\star}$ is Butler as a pure subgroup of the Butler group $C$. We infer $(B, a\rangle_{\star}=$ $B+C_{1}+\ldots+C_{m}$ with $C_{i}$ of rank 1 in $A^{\prime}$. Lemma 1 implies (i).

To verify (ii), let $D / B$ be a finite rank pure subgroup of $A / B$. By definition, there exist a finite number of rank 1 subgroups $A_{i}$ of $A$ such that $B+A_{1}+\ldots+A_{k}=E$ is pure in $A$ and contains (a maximal independent set of $D$ and hence) $D$. Evidently $E / B$ is Butler, and so is its pure subgroup $D / B$.

Conversely, let (i) and (ii) be satisfied. Given a finite subset $S$ of $A$, let $D=$ $\langle B, S\rangle_{\star}$. By (ii), $D / B=D_{1} / B+\ldots+D_{n} / B$ for rank 1 pure subgroups $D_{i} / B$ of $D / B$. In view of (i), each $D_{i}$ is of the form $D_{i}=B+A_{i 1}+\ldots+A_{i k_{i}}$ with $A_{i j}$ of rank 1. Thus $D=B+\sum_{i} \sum_{j} A_{i j}$ contains $S$ and it is as desired. We conclude that $B$ is decent in $A$.

Added in Proof Dr A. Giovannitti has called our attention to the paper "Butler groups, valuated vector spaces and duality" Rend. Sem. Mat. Univ. Padova, 72, (1984), 13-19, by F. Richman who introduced the concept of semi-balancedness (which is equivalent to our prebalancedness) and proved our Theorem 5 .

\section{REFERENCES}

[1] U. Albrecht and P. Hill, 'Butler groups of infinite rank and Axiom 3', Czechoslovak Math. J. 37 (1987), 293-309.

[2] D.M. Arnold, Finite Rank Torsion-free Abelian Groups and Rings, Lecture Notes in Math. 931 (Springer-Verlag, Berlin, Heidelberg, New York, 1982).

[3] L. Fuchs, Infinite Abelian Groups, Vol. 1-2 (Academic Press, New York, 1970 and 1973).

[4] A. Giovannitti, 'Extensions of Butler groups', in Abelian Group Theory, Editors R. Göbel, L. Lady and A. Madz, Lecture Notes in Math, 1006, pp. 164-170 (Springer-Verlag, Berlin, Heidelberg, New York, 1983).

[5] R.B. Warfield, 'Extensions of torsion-free abelian groups of finite rank', Arch. Math. 23 (1972), 145-150.

Department of Mathematics

Tulane University

New Orleans LA 70118

United States of America
Department of Mathematics

University of Orange Free State

Bloemfontein 9300

Republic of South Africa 